\title{
Scanning both Hips and the Lumbar Vertebrae in Men Identifies More Patients with Osteoporosis than Scanning One Hip and the Lumbar Vertebrae
}

\author{
Ronald C Hamdy ${ }^{1 *}$, Miriam M Mottl ${ }^{2}$, Matthew Perdue ${ }^{1}$, Isaac Cline ${ }^{1}$ and Yali Liu ${ }^{3}$ \\ ${ }^{1}$ James H. Quillen College of Medicine, East Tennessee State University, USA \\ ${ }^{2}$ University of Rostock Medical School, Rostock, Germany \\ ${ }^{3}$ ETSU Department of Mathematics and Statistics, East Tennessee State University, USA
}

\begin{abstract}
Background: In the absence of fragility fractures, the diagnosis of osteoporosis is established by bone densitometry: a T-score of -2.5 or lower in the femoral neck, total hip or lumbar vertebrae. One hip and the lumbar vertebrae are routinely scanned, and there is no consensus which hip should be used. The purpose of this retrospective study is to determine whether, in a male population, scanning both hips and the lumbar vertebrae identifies more patients with osteoporosis than scanning only one hip and the lumbar vertebrae.
\end{abstract}

Methods: We retrieved data from 1,048 male Caucasian patients referred to our Center who were not on treatment for osteoporosis, had no documented bone pathology and had interpretable scans of both hips and the lumbar vertebrae.

Results: More men aged 80 years and older were diagnosed with osteoporosis when scans of both hips and the lumbar vertebrae were considered, compared to the left hip and lumbar vertebrae $(7 \%)$ or right hip and lumbar vertebrae $(6 \%)$. The differences in diagnostic categories were less pronounced in younger subjects: only $2 \%$ more men younger than 60 years were diagnosed with osteoporosis when both hips and the lumbar vertebrae were scanned compared to just one hip and the lumbar vertebrae.

Conclusions: We recommend that in Caucasian men, especially those aged 80 years and older, both hips be scanned in addition to the lumbar vertebrae.

Keywords: Osteoporosis; Diagnostic imaging; Dual X-ray absorptiometry

\section{Introduction}

The diagnosis of osteoporosis, in the absence of fragility fractures, is established by Dual X-ray Absorptiometry (DXA). The World Health Organization (WHO) criteria are universally used: if the T-score at the proximal femur (femoral neck or total hip region) or lumbar vertebrae is -2.5 or lower, the diagnosis is osteoporosis.

There are, however, no guidelines as to which hip, or whether one or both hips should be scanned in addition to the lumbar vertebrae. Arguments for scanning both hips include the differences between the left and right T-scores observed in some patients who may result in the patient being classified differently if one or both hips are scanned [1-5], and being able to follow up patients who fracture one hip or undergo hip replacement. Arguments against scanning both hips include the good correlation that exists between the BMD of both hips [6-11], unnecessary exposure to radiation and the time taken to perform the scan. On the other hand, exposure to radiation is now so small as to be negligible, and the time factor is no longer an issue with modern densitometers, many offer the ability of scanning both hips without repositioning the patient.

In practice, which hip is scanned depends on the physical position of the densitometer in the scanning room and the technologist's preference. In our Center we routinely scan both hips, in addition to the lumbar vertebrae, and use the lowest T-score to make the diagnosis. We do not average both hips. It has been our clinical impression that some patients will be classified differently if the scan includes both hips rather than one hip. We have previously reported these findings in women [1].

The purpose of this retrospective study is to determine, in a male population, the diagnostic yield of scanning both hips, rather than just one hip, in addition to the lumbar vertebrae. We restricted our study to Caucasian males to increase the homogeneity of the group.

\section{Materials and Methods}

In this retrospective study, we examined the clinical records of Caucasian male patients referred to our Center, who were not treated for osteoporosis, had no documented bone pathology, and had the upper four lumbar vertebrae and both hips scanned during the same session using the Hologic Delphi (software versions 11.1 and 11.2) densitometer. Left and right hips were scanned separately. No scan was acquired in the automated bilateral scan mode. Only patients who had interpretable DXA scans of both hips and the upper four lumbar vertebrae were included in the study. The BMD and T-score values of the upper four lumbar vertebrae, the femoral neck, and total hip region of each hip were retrieved. The study had been approved by the Institutional Review Board.

T-scores were calculated for the upper four lumbar vertebrae,

*Corresponding author: Ronald C Hamdy, James H. Quillen College of Medicine East Tennessee State University, P.O. Box 70429, Johnson City, TN 37614, USA, Tel: 1-423-439-8830; Fax: 1-423-979-3438; E-mail: hamdy@etsu.edu

Received January 31, 2013; Accepted April 27, 2013; Published May 03, 2013

Citation: Hamdy RC, Mottl MM, Perdue M, Cline I, Liu Y (2013) Scanning both Hips and the Lumbar Vertebrae in Men Identifies More Patients with Osteoporosis than Scanning One Hip and the Lumbar Vertebrae. Aging Sci 1: 103. doi:10.4172/2329-8847.1000103

Copyright: @ 2013 Hamdy RC, et al. This is an open-access article distributed under the terms of the Creative Commons Attribution License, which permits unrestricted use, distribution, and reproduction in any medium, provided the original author and source are credited. 
femoral neck and total hip regions from the standard Hologic reference data (manufacturer-specific spine database and National Health and Nutrition Examination Survey [NHANES] III for the hip). We used the WHO diagnostic classification [12], to classify patients into the diagnostic categories: osteoporosis (T-score-2.5 or lower), osteopenia (T-score lower than-1.0, and higher than-2.5), or normal (T-score-1.0 or higher). Precision measurement was calculated based on duplicate scans in 30 patients with repositioning between the scans.

We calculated the number of patients in each diagnostic category and compared the results when the lumbar vertebrae and either hip were taken into account to those when the lumbar vertebrae and both hips were considered. We then excluded patients who had osteoporosis of the lumbar vertebrae because in these patients the T-scores of the hip would not alter the final diagnosis. We looked at our population as a whole and then at various age groups.

McNemar's symmetry test for paired observations was used to determine proportional differences in the diagnostic classification of the various scan sites. Differences were considered significant for all tests at $\mathrm{P}<0.05$. We calculated the Pearson correlation coefficients of the BMD of the total hip and femoral neck for the right and left hip. Using the two-sample proportions test we examined the left and right hip differences in BMD at each site and compared these differences to the Least Significant Change (LSC) of our Center and the extent to which they affect the diagnostic classification. We used the paired t-test to determine whether there were any significant differences between the BMD of the left and right hip.

\section{Discussion}

We examined the records of 1,823 male patients referred to our Center and retrieved data from 1,048 who satisfied our inclusion criteria. Their mean age was 66.5 years (SD 11.3). The site-specific LSC calculated at the $95 \%$ confidence interval were: 0.015 for right total hip, 0.038 for right femoral neck, 0.017 for left total hip, 0.039 for left femoral neck, and $0.023 \mathrm{~g} / \mathrm{cm}^{2}$ for the lumbar vertebrae. All patients were scanned by the same technologist.

Based on the WHO diagnostic classification, there were no significant differences $(\mathrm{p}=0.404)$ when the lowest T-score of the lumbar vertebrae and right or left hip (femoral neck or total hip regions) is taken into account: 25\% (left hip) and 26\% (right hip) of the patients were classified as having osteoporosis. However, the proportion of patients in the various WHO diagnostic categories changed significantly $(\mathrm{P}<0.0001)$, when both hips in addition to the lumbar vertebrae were considered: compared to either one hip and lumbar vertebrae: more patients (29\%) were diagnosed with osteoporosis. The percentages of patients in each diagnostic category in various age groups are shown in figure 1. Whereas in those aged 80 years and older there were differences of 6 to $7 \%$ between those diagnosed with osteoporosis when both hips and lumbar vertebrae were scanned versus the right or left hip, (in addition to lumbar vertebrae) respectively; in those under the age of 60 years, the differences were $2 \%$.

In our series of 1048 patients, 130 had evidence of osteoporosis in the lumbar vertebrae. We excluded these from the subsequent analysis as in these patients the results of the hip scans would not alter the diagnostic category. Of the remaining 918 patients whose T-scores of the lumbar vertebrae were in the osteopenic or normal range, 176 patients (19\%) had a T-score of -2.5 or lower in either the left or right femoral neck or total hip; 105 (11\%) had a T-score of -2.5 or lower on both sides (femoral neck or total hip); 40 (4\%) had a T-score in the osteoporotic range (<-2.5 or lower) in the right (but not left hip) and 31 (3\%) had a T-score in the osteoporotic range in the left, but not right hip.

As expected, there were strong positive correlations between the $\mathrm{BMD}$ of the left and right femoral neck $(\mathrm{r}=0.924)$ and left and right total hip ( $\mathrm{r}=0.918)$.

\section{Results}

Results of this study show that although there is a good correlation

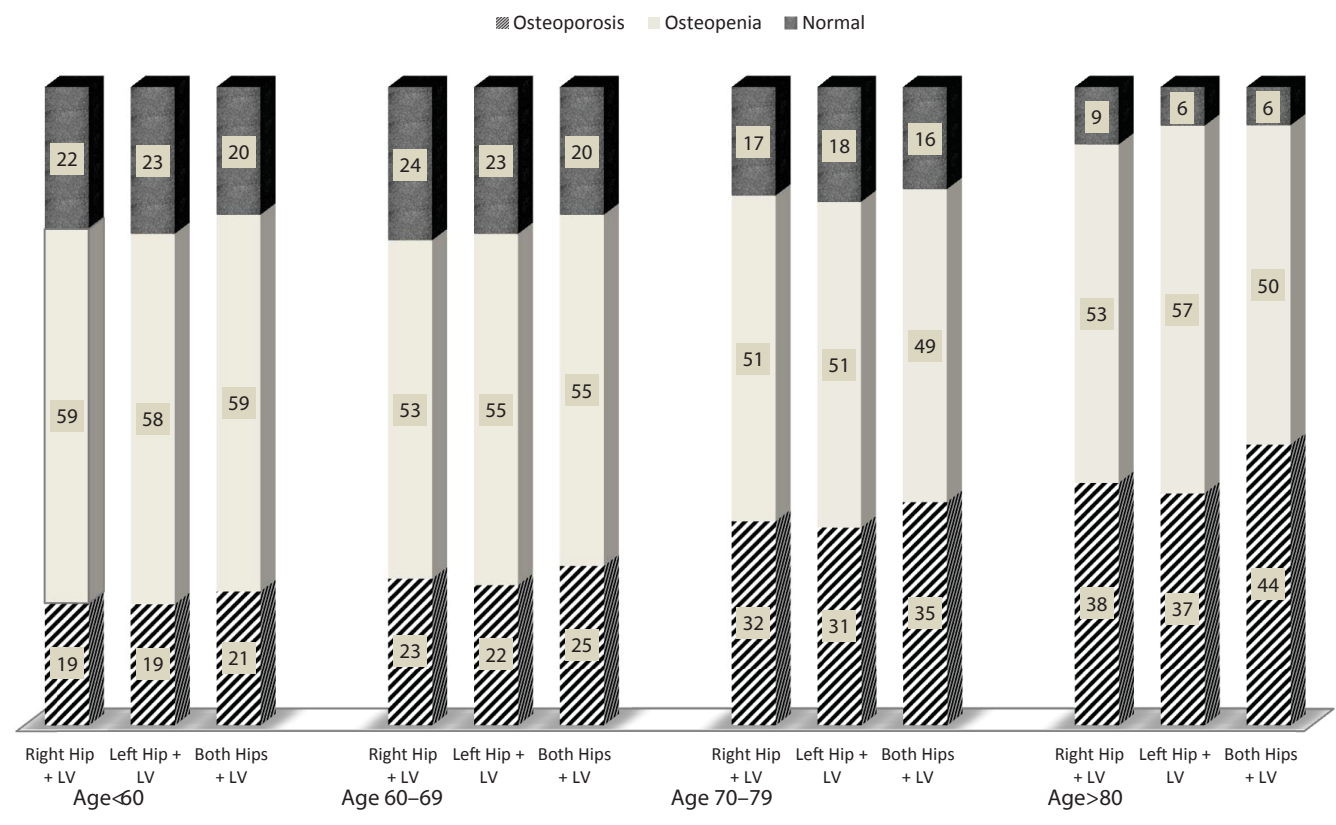

Figure 1: Percentage of patients in each diagnostic category in various age groups, using different combinations of sites. 
Citation: Hamdy RC, Mottl MM, Perdue M, Cline I, Liu Y (2013) Scanning both Hips and the Lumbar Vertebrae in Men Identifies More Patients with Osteoporosis than Scanning One Hip and the Lumbar Vertebrae. Aging Sci 1: 103. doi:10.4172/2329-8847.1000103

between the BMD of the right and left femoral neck and total hip, the differences in T-scores are of sufficient magnitude to change the patient's diagnostic classification. In the population aged 80 years and older more patients are diagnosed with osteoporosis if, in addition to the lumbar vertebrae, both hips are considered $(44 \%$ of the study population) rather than only one hip: 37\% (left hip) and 38\% (right hip). These results are in agreement with previously published work on women [1].

As there is no significant difference in the number of patients diagnosed with osteoporosis if either hip is taken into account, it is probable that each hip identifies different patients with osteoporosis, suggesting that although there is a very good correlation between the BMD of the femoral neck of both sides $(r=0.924)$ and between the BMD of the total hip of both sides ( $r=0.918)$, the differences in BMD between the two sides are of sufficient magnitude to change the T-scores and hence diagnostic classification. Including the lumbar vertebrae does not attenuate the overall differences in diagnostic classification when both hips are considered versus either the left or right hip. Therefore, scanning both hips, in addition to the lumbar vertebrae will classify more patients with osteoporosis than scanning one hip in addition to the lumbar vertebrae. Although this is intuitive: the more sites are scanned the more likely is one site to be -2.5 or lower, results of our study provide evidence to this effect. This also suggests that when both hips are scanned, the lowest T-score rather than the mean T-score should be taken into consideration for diagnostic purposes.

Given the relatively short time needed to scan each hip, this additional scan should not impact on the overall time needed to scan patients; in fact many modern densitometers routinely scan both hips. These results are in agreement with other published work showing that significantly more women will be diagnosed with osteoporosis if both hips, in addition to the lumbar vertebrae, are considered compared to just one hip and the lumbar vertebrae [1].

The availability of the Vertebral Fracture Assessment (VFA) tool may lower the returns of scanning both hips, as the diagnosis of osteoporosis can be established in the presence of fragility vertebral compression fractures. On the other hand, VFA requires an additional component to the densitometer, and often the patient has to be repositioned. In most instances it is quicker to scan the contra lateral hip than to perform the VFA.

Similarly, the availability of the Fracture Risk Assessment Tool $\left(\right.$ FRAX $\left.^{\circ}\right)[12]$ may lower the returns of scanning both hips: if the 10year probability of sustaining a fracture exceeds the recommended threshold for treatment, it may not be necessary to scan both hips. Although the FRAX" tool is incorporated in some densitometers and is easily accessible, free of charge, on the internet; it entails gathering data and is more time consuming than scanning the other hip.

A relative weakness of our study is the relatively small number of patients in each age category: 139 (aged 80 years and older), 279 (aged 70-79 years), 358 (aged 60-69 years) and 272 (under age 60 years). Notwithstanding, our recommendation is that in men, especially older men, both hips be scanned in addition to the lumbar vertebrae to maximize the number of patients diagnosed with osteoporosis.
Scanning both hips also offers the advantage of being able to monitor the bone mass even after the patient sustains a hip fracture.

\section{Conclusion}

Our study shows that in Caucasian males, especially older ones, although there is a good correlation between the BMD and T-scores of the right and left hip, scanning both hips in addition to the lumbar vertebrae identifies more patients with osteoporosis than scanning just one hip and the lumbar vertebrae. We recommend that both hips be routinely scanned especially in older Caucasian men.

\section{References}

1. Hamdy RC, Kiebzak GM, Seier E, Watts NB (2006) The prevalence of significant left-right differences in hip bone mineral density. Osteoporos Int 17: 1772-1780.

2. Mazess RB, Nord RH, Hanson JA, Barden HS (2000) Bilateral measurement of femoral bone mineral density. J Clin Densitom 3:133-140.

3. Rao AD, Reddy S, Rao DS (2000) Is there a difference between right and left femoral bone density? J Clin Densitom 3: 57-61.

4. Wong JCH, McEwan L, Lee N, Griffiths, Pocock NA (2003) The diagnostic role of dual femur bone density measurement in low impact fractures. Osteoporo sint 14: 339-344

5. Petley GW, Taylor PA, Murrills AJ, Dennison E, Pearson G, et al. (2000) An investigation of the diagnostic values of bilateral femoral neck bone mineral density measurements. Osteoporos int 11: 675-679.

6. Lessig HJ, Metzer MS, Siegel JA (1987) The symmetry of hip bone minera density: a dual photon absorptiometry approach. Clin Nucl Med 12: 811-812.

7. Lilley J, Walters BG, Heath DA, Drolc Z (1992) Comparison and investigation of bone mineral density in opposing femora by dual $\mathrm{x}$-ray absorptiometry. Osteoporos Int 2: 274-278.

8. Faulkner KG, Genant HK, McClung M (1995) Bilateral comparison of femora bone density and hip axis length from single and fan beam DXA scans. Calcif Tissue Int 56: 26-31.

9. Bonnick SL, Nichols DL, Sanborn CF, Payne SA, Moen SM, et al. (1996) Right and left proximal femur analyses. Is there a need to do both? Calcif Tissue Int 58: $307-310$.

10. Franck H, Munz M, Scherrer M (1997) Bone mineral density of opposing hips using dual energy $\mathrm{x}$-ray absorptiometry in single beam and fan-beam design. Calcif Tissue Int 61: 445-447.

11. Yang R, Tsai K, Chieng P, Liu T (1997) Symmetry of bone mineral density at the proximal femur with emphasis on the effect of side dominance. Calcify Tissue Int 61: 189-191.

12. Kanis JA (2007) Assessment of osteoporosis at the primary care level. Technica report. University of Sheffield, UK: WHO Collaborating Center. 